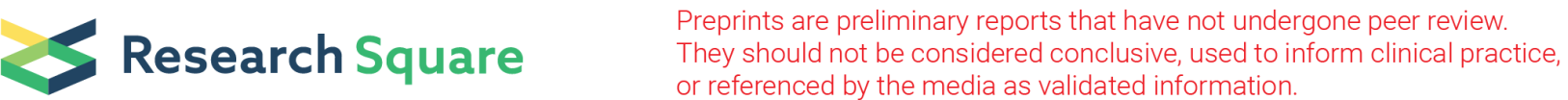

\section{Improving the control ability of pulmonary tuberculosis with an internet based eDOTS system in the communities of Kashgar and Urumqi, Xinjiang of China}

\section{Gang Guo}

First Affiliated Hospital of Xinjiang Medical University

\section{Li Sun}

Yingjisha County Center for Disease Control and Prevention

\section{Qimanguli Wushouer}

First Affiliated Hospital of Xinjiang Medical University

\section{Bin Jia}

First Affiliated Hospital of Xinjiang Medical University

\section{Muladier Yusufu \\ Kashgar Region Lung Hospital}

\section{Shu Wen}

Kashgar Region Lung Hospital

\section{Tuerhong Abudureyimu}

Kashi Region Lung Hospital

\section{Xiaowang Peng}

Kashigar Region Lung Hospital

\section{Zhenjiang Liu}

Kashgar Region Lung Hospital

\section{Xirizat Mamuti}

Kashigar Region Lung Hospital

\section{Yanggui Chen}

Urumqi Centers for Disease Prevention and Control

Jian Zhang

High-tech District Center For Disease Control and Prevention of Urumqi

\section{Yuling Yang}

Xinhuarui Information Co., LTD

\section{Liusheng Huangfu}

Xinhuarui Information Co., LTD

Jun Li

First Affiliated Hospital of Xinjiang Medical University 
Wenbao Zhang ( $\nabla$ wenbaozhang2013@163.com)

Xinjiang Medical University

\section{Research Article}

Keywords: Pulmonary tuberculosis, DOTS, eDOTS, Control, Xinjiang, China

Posted Date: May 17th, 2020

DOI: https://doi.org/10.21203/rs.3.rs-28641/v1

License: (c) (1) This work is licensed under a Creative Commons Attribution 4.0 International License. Read Full License 


\section{Abstract}

Background: Although the direct observed treatment strategy (DOTS) has been used for the control of pulmonary tuberculosis (PTB) in Xinjiang Uygur Autonomous Region for more than 30 years, the disease is still highly endemic in the region. To increase ability of PTB treatment and control efficacy, we developed a new eDOTS system for reminding, monitoring and educating PTB patients for regularly taking antibiotics.

Methods: A total 167 new cases with active PTB from Kashgar villages and Urumqi City in Xinjiang were involved in the study. Among them, 81 patients were from villages with 43 patients using eDOTS system and 38 patients with traditional DOTS as control. Eighty six patients were from township with 50 patients using eDOTS and 36 patients using DOTS.

Findings: PTB patients from villages in Kashgar treated with DOTS showed low compliance of taking antibiotics, only $27.9 \%$ the patients took medicine consistently once the network service for eDOTS system was removed. However, with the help of village and hospital doctors through the eDOTS system, the medical compliance were increased to $70-85 \%$. The treatment compliance with eDOTS of patients from township (100\%) was significantly higher than that of those patients from the rural villages $(49 \%)$ in the first 6 weeks ( $X 2=33.3, P<0.01$ ). DR X-ray Scores of PTB lesions showed that the patients using eDOTS either from the villages or from township significantly decreased their lesion score points compared to these scores from those patients using DOTS by 1.81 points $₫ 95 \% \mathrm{Cl}(0.72-2.90)$ and 1.05 points $\varangle 95 \% \mathrm{Cl}(0.15-1.95)$ after 25 weeks of treatment, respectively. In the township, those 50 patients were looked after by one community doctor with eDOTS.

Conclusion: eDOTS is an effective means of treating PTB patients in terms of reducing doctor's work and increasing the compliance of taking antibiotics regularly during the treatment course. One doctor can easily look after 50 or more PTB patients through the eDOTS system. The constantly education program online through the system for the patients especially in villages plays an important role in encouraging TB patients taking antibiotics for a long period.

\section{Background}

Pulmonary tuberculosis (PTB) is a serious public health disease and challenges the population health system in the world, especially in China, accounting for 15 percent of the global TB burden ${ }^{[1-3]}$. According to a WHO report 2018, China accounts for 8.9 percent of the world's new TB cases each year ${ }^{[4]}$. Xinjiang Uygur Autonomous Region (XUAR) is a high endemic area of TB in China ${ }^{[5-8]}$. According to the fifth national TB epidemiological survey in 2010, the prevalence of active TB in Xinjiang was 1526/100, 000 residents and the prevalence of smear-positive TB was 196/100, 000, which the figures were 3.32 times and 2.97 times higher than the national average incidence, respectively. The reported incidence of southern XUAR was 610.33 / 100,000 which was 11.3 times the national average and that in the local four prefectures (Kashgar, Hotan, Aksu and Kizilsu Kirghiz) accounts for $70 \%$ of the total TB 
patients in the region ${ }^{[6]}$. A total of 71, 427 TB patients were registered in XUAR in $2018^{[7]}$, with a reported incidence of 292.3/ 100, 000 ranking first in China, which was 4.8 times the national average (61/ $100,000)^{[9]}$. From 2008 to 2018 , the highest annual average reported incidence of tuberculosis in XUAR was found in Yingjisha county of Kashgar $(720.56 / 100,000)$, which was 25 times higher than Urumqi city $(28.44 / 100,000)^{[7]}$.

WHO has recommended the direct observed treatment strategy (DOTS) ${ }^{[10]}$ for stopping TB transmission for decades and the strategy has been introduced into China since 1990's, which had reduced half of TB prevalence by $2010^{[11]}$. However, the strategy did not effectively work in XUAR. In contrast, a survey performed in 2010-2011 showed the prevalence $(1526 / 100,000)$ was almost the same to that $(1570 / 100,000)$ in $1979^{[6]}$, and 2.29 folds higher than the prevalence in 1990(666/100,000). The main reason was that the compliance of TB patients for taking antibiotics was not as high as designed ${ }^{[12]}$.

To increase the patient's compliance, we developed a new DOTS system which is based on mobile network technology, named as eDOTS (electronic DOTS). The system was composed of several parts including an intelligent electronic medicine kit (eBox) for a patient, network and mobile connecting system, a central processing platform designed for the Centers for Disease Control and Prevention (CDC) and mobile APP and/or Wechat (software for all persons involved in the control and treatment program) (Fig. 1). In the present study, we showed the usage of the eDOTS in treatment of TB patients,which significantly increased treatment efficacy against TB.

\section{Methods}

\section{Patients and treatment management}

For testing the eDOTS system used in villages, we selected Yinjisha County of Kashgar Prefecture in southern XUAR. Two communities with $95 \%$ of villager being Chinese Uygur were selected for respectively using DOTS and eDOTS. Considering the cross impacting between villages with the two treatments, the distance of villages with different managements was at least $10 \mathrm{~km}$ apart from each other. One villager doctor looked after the whole PTB patients in a village with a total 13 villages involved. For testing the treatment management in township, we selected TB patients from residential communities in Urumqi city area $₫$ where one community doctor was in charge of a community.

PTB patients aged between 15 and 75 were randomly selected by the timing of diagnosis. Pregnant women and patients with HIV infection or using hormone were excluded. Most of the PTB patients were diagnosed through annual health check program referring to the criteria ${ }^{[13]}$ published by the Ministry of Health of China. All the patients showed DR (Digital Radiography) positive with clear and typical symptoms such as night sweats, chest pain, persistent cough and fever for two weeks with some coughing up blood sputum. In addition, we established a DR scoring system for evaluating the TB status mainly according to DR chest radiograph. TB lesion image features were classified into three levels based on lesion image features including exudation areas (3 scores), pathology including proliferation (4 
scores) and infiltration and density of TB lesion (3 scores) (Supplementary data 1). Three X-ray experts were asked to score the TB lesion progress for each of the patients. Examples are shown in Fig. S1 and S2.

The study was reviewed and approved by the Ethic Committee of the First Affiliated Hospital of Xinjiang Medical University with approval No. (20150824-01). All the patients involved in the project had an informed consent signed.

\section{Training plan}

All doctors from villages and downtown communities and staff involved in the study had one-day training for introduction and use of the device and the system. Once a PTB patient was diagnosed and confirmed, he or she was registered as a TB patient in the study and recorded all possible information for DOTS treatment including name, ID, age, gender, family location, diagnosis information such as smear results, X-ray and treatment prescription etc. After registered, an eDOTS box and a week of anti-TB medicine were supplied. Doctors saw their patients once a week and added the drugs for the next week.

\section{Drug treatment}

Standard anti-TB drug combinations containing isoniazid $(H)$, rifampicin $(R)$, pyrazinamide $(\mathrm{Z})$, ethambutol (E) were applied for those PTB patients according to WHO recommendation as the first line anti-TB drugs ${ }^{[14]}$. Two treatment courses for all the cases include a two-month intensive phase with a daily dose of fixed-dose combination tablets containing HRZE, followed by a four-month continuation phase of daily HR ${ }^{[15]}$.

\section{Data records}

The eDOTS system recorded the patient's medication activity and automatically transferred into the management system in CDC. If a patient did not take the medicine in time, the system automatically transferred a message to her/his doctor and relative asking them to remind the patient taking the medicine. If the patient was still not taking the medicine after $5 \mathrm{pm}$ in the day, the system recorded as "non-taking medicine". In DOTS group, doctors were normally ticking on the book for taking or not taking medicine. Results

\section{Intervention treatment}

A total of 167 active TB patients were registered from 14 villages from 2 communities in Yinjisha County of Kashgar and 2 districts in Urumqi city, XUAR. Ninety-three patients were treated with eDOTS and 74 with DOTS. In eDOTS group, only 3 patients fully and completely took the treatment during the treatment course, that was $8 \%$ of the total patients after 6 months of treatment course. The most patients did not fully take their medicine for some of reasons including neglect of medication, drug side effect, visiting friends. However, the doctors responsible for DOTS in both rural and downtown area all claimed that $100 \%$ of all 74 enrolled cases were on medication every day during the intervention. 


\section{Treatment in the villages}

Eight-one patients (Uygur ethnic) from villages were enrolled in a time course of being diagnosed with 43 patients from 9 villages treated with eDOTS system and 38 patients from 5 villages using DOTS as control.

In the first 6 weeks of management in the eDOTS group, the average medication compliance rate was $48.8 \%, 95 \% \mathrm{Cl}(46.5 \%, 51.1 \%)$. At week 7 , the network was accidently disconnected and DOTS had been supplied for those eDOTS patients for two weeks. However, when the system was reused, the compliance rate was dropped to $27.9 \%, 95 \% \mathrm{Cl}(22.8,33.0 \%)$. We then asked the village doctors to closely contact with those patients and the rate for taking drugs was increased to $65.1 \%, 95 \% \mathrm{Cl}(59.7 \%, 70.5 \%)$ at week 20 subsequently. After we then asked a well trained doctor from a hospital to contact with those patients who did not take drugs, the average medication compliance rate was increased to $77.2 \%, 95 \% \mathrm{Cl}(75.1$, 79.3\%) (Fig. 2).

\section{Treatment in the township}

We used the eDOTS for 50 patients (Han ethnic 34, Hui ethnic 16) compared with 36 patients (Han ethnic 28 , Hui ethnic 8) using DOTS in Urumqi city. Those 50 patients were monitored by one community doctor with his mobile phone or laptop through the system.

In the first 12 weeks of the intervention, the compliance rate of the patients with eDOTS was almost $100 \%$. After 12 weeks, the rate was slightly dropped, but the overall compliance rate was kept in more than 80 percent (Fig. 3).

The first 6 weeks of treatment of patients with eDOTS showed that village patients had $45-55 \%$ of compliance taking anti-TB drugs, which was significantly lower than the compliance rate of patients in township $(99-100 \%)\left(X^{2}=193.445, P<0.01\right)$.

\section{DR X-ray score analysis}

There was no statistic difference on TB scores from patients between town and villages which were both $9.1 \pm 1.3$ points before treatment. Whether DOTS or eDOTS was used, TB scores can be significantly reduced in both downtown and rural area at the end of the intervention period (Fig. 4). We analyze the scores by using two-way ANOVA with Tukey's multiple comparisons test after 25-26 weeks of treatment. TB score (3.5 \pm 0.6 points) of patients from Urumqi with eDOTS was significantly lower than those (4.5 \pm 1.6$)$ with DOTS by 1.1 points [ $95 \% \mathrm{Cl}(0.2,2.0), q=5.036, p=0.05<0.01]$. In village, TB score $(4.2 \pm 0.6$ points) of patients with eDOTS was lower significantly than those $(6.0 \pm 1.6)$ with DOTS by 1.8 points $[95 \% \mathrm{Cl}(0.5,3.1), q=6.052, p<0.01]$. Comparison after using eDOTS, there was no difference between the score (3.5 \pm 0.6 points) of TB patients in downtown and those ( $4.2 \pm 1.2$ points) in rural areas ( $q=2.596$, $p>0.05)$. Comparison only after using DOTS, the TB score (4.5 \pm 1.6 points) of patients in downtown was 
lower significantly than those $(6.00 \pm 1.5$ points) in village by 1.5 points $[95 \% \mathrm{Cl}(2.5,0.5), q=6.339$, $p<0.01)]$.

\section{Discussion}

Data from China's fifth national tuberculosis epidemiological sampling survey showed that only $14.4 \%$ of active tuberculosis patients were smear-positive ${ }^{[15]}$. In addition, it is well known that once patients take the medicine for few days, some cases will become smear negative. Therefore, sputum smear was not used as a parameter for evaluating the treatment efficacy for the whole time course of treatment in our study. The change in DR is a reliable method for identifying the active or inactive PTB, and we scored and compared the DR scores of those patients before and after treatment. We used two parameters, compliance rate and TB DR score changes to compare the efficacy of both systems used in villages and township.

\section{The system of eDOTS is an effective tool for increasing TB treatment capacity}

TB Patients generally need combined-drug treatment with a long period of 6-8 months ${ }^{[16]} \rrbracket$ so taking medicine compliance and re-examination are the keys to TB cure ${ }^{[16-17]}$. Failure to compliance can lead to prolonged disease duration, drug resistance, relapse and refractory conditions ${ }^{[18-19]}$. Although the international TB control strategy- DOTS was introduced to improve the cure of TB囚TB control programmes are not fully functioned as required by the guidelines in the remote and impoverished areas of China ${ }^{[19]}$. Some surveys conducted in recent years have shown that treatment compliance among TB patients is not ideal in China with DOTS management ${ }^{[19-21]}$. In our study, the rate of taking drug was just $48.8 \%$ in the villages after internet off at week 6 , which may reflect the real treatment compliance rate of village patients by using DOTS as the book marks being always $100 \%$ could not reflect the real compliance for taking anti-TB medicine. We believe the low of compliance for taking drugs is the key for the high prevalence of TB in rural area of XUAR, comparing those in Jiangsu (89\%), Anhui (90.5\%), and Ningxia (93.3\%) of China ${ }^{\text {[21]. }}$

The developed eDOTS system for promoting compliance may meet the need of control TB in high endemic areas in Xinjiang as the traditional DOTS was not successful in the region. Through the comparison, we found that whether using eDOTS in urban or rural areas showed better therapeutic efficacy than those patients using the DOTS system alone.

\section{The system of eDOTS solved the problems from DOTS carried out in Xinjiang}

From our views, we believe the problems from DOTS are including:

\section{No proper reminding}

The problems for using DOTS including no proper reminding in time for taking drugs. Only with DOTS, the patients could not complete their treatment course due simply to forgetting taking medicine very often, 
strongly suggesting they need a reminding advice. While, the feature of the eDOTS system includes an eBox can automatically remind the patient taking medicine every day according to convenient time for each individual daily.

\section{No convenient health educational measures}

In our study, after the village doctors and hospital doctors intervened at week 13 and 21 successively, the compliance rate increased to $65.1 \%$ and $77.2 \%$ respectively. This indicates that regularly communication of doctors with their patients is very importance for encouraging the TB patients taking medicine. According to the DR score analysis, the treatment status of the patients in township using DOTS was better than that of rural patients, indicating that the compliance of rural patients was lower than that of town patients. It also showed that the compliance rate for taking antibiotics in these villages was lower than those patients in the city according to the results recorded by eDOTS. Inability to recognize the significance of long-term treatment caused by a low level of knowledge and not following doctor's orders

were the primary reasons of poor compliance to treatment of patients in rural ${ }^{[22-25]}$. DOTS has not played its due role in improving patient compliance through health education in XUAR, especially in rural. While, the eDOTS box has a key to link the patient and doctor. Firstly, once the doctor has the message, she or he can communicate with the patients for answering the questions from the patients. Secondly, the eDOTS system has a voice education program and TB patients can hear from the advice every day. The voice education program contains 180 key questions about the treatment of TB by taking antibiotics and is arranged mainly according to the treatment condition and most questions, which would be asked by the patients in that time course of treatment. Lack of proper monitoring system

During the first 6 weeks of application in the rural eDOTS group, the average patient compliance rate was $48.8 \%$. At the seventh week, the eDOTS internet was disconnected and all the patients were asked to take medicine as DOTS. However, when the network was connected at week 8 , the eDOTS recorded data shows that the patient medication compliance rate was $27.9 \%$. We can speculate that the compliance rate of rural TB patients managed with normal DOTS was likely at this level of compliance for taking medicine. We often couldn't get objective data on the activity of TB treatment with DOTS. While eDOTS was used, once the medicine box was open, the system was activated immediately and recorded the taking drug process automatically.

\section{More PTB patients are needed to be looked after}

XUAR has a heavy burden of tuberculosis, with higher prevalence in rural areas than in urban areas, higher prevalence in southern XUAR than in northern and eastern XUAR. According to the results of the fifth national epidemiological survey on tuberculosis in 2010, the prevalence rate of active tuberculosis among people over 15 years old only in kashgar is $2,727 / 100,000$ (about 109,000 patients, in all ) [3], which requires a large number of doctors to manage. The patients need an easy way to be reminded taking medicine, and doctors and the families of patients need a system to monitor the patients taking medicine. In addition, TB patients need medical and psychological consultation from doctors and other 
society workers especially when they have difficulty and problem for taking the medicine. Although it is the duty for village doctors to face to face check patients taking medicine every day in the DOTS strategy, most of the doctors could not reach all the patient's houses every day due simply to so many patients scattered. While the eDOTS system allows doctors manage more TB patients, with which one doctor can easily look after 15 patients or even more in village, and more than 50 patients in township. Especially in rural areas, where patients have limited transportation, the use of eDOTS can more effectively reduce the workload of village doctors while improving patient treatment compliance.

\section{Conclusion}

The application of the eDOTS system will be an effective measure to implement the WTO strategy to "stop TB" ${ }^{[26]}$ and it is an efficient system in terms of reminding and recording PTB patients taking medicine and doctor's activities for looking after their patients. The system may also increase doctor's management ability and improve treatment efficiency significantly in terms of reducing the visiting times of village/community doctor to their patients.

In summary, eDOTS can improve treatment compliance and TB cure rates of patients effectively by providing reminder services and health education to patients conveniently and by providing more scientific monitoring measures and more efficient patient management solution to medical staffs or the governments.

\section{Abbreviations}

\section{DOTS}

the traditional direct observed treatment strategy, eDOTS:the eletronic direct observed treatment strategy, DR:digital radiography, PTB:pulmonary tuberculosis, XUAR:Xinjiang Uygur Autonomous Region

\section{Declarations}

\section{Acknowledgements}

Thanks to these doctors for their expertise in establishing of TB DR score criteria. Bin Jia, Qimanguli Wushouer, Wenya Liu, Jin Wang, Muhebaiti Mehesuti and Astar Wushour for establishing the classification of TB X-ray chest image and scored the TB lesion progress

\section{Funding}

This study was financially supported by WHO-TDR small grant (201344525) and Research Program of First Affiliated Hospital of Xinjiang Medical University (SB-2015-02).

\section{Availability of data and materials}

All the data supporting our findings have been presented in this paper. 


\section{Authors' contributions}

GG and WZ was the principal writer of the proposals and WZ designed the protocol of investigation and evaluation. GG and LH coordinated the fields survey and data record. LS, MY, SW, TA, XP, ZL, YC and LZ collected, confirmed TB samples and applied eDOTS system. LH and YY developed and improved the eDOTS system. JL and GG collected the data and finalised the statistical analysis. WZ took part in the analyses, interpretation, and critically revised the paper. All authors read and approved the final paper. All authors read and approved the final manuscript.

\section{Ethics approval and consent to participate}

The study was reviewed and approved by the Ethical Committee of the First Affiliated Hospital of Xinjiang Medical University and in compliance with medical ethics. Informed consents were obtained from human participants of this study.

\section{Consent for publication}

Not applicable.

\section{Competing interests}

The authors declare that they have no competing interests.

\section{Author details}

1. State Key Laboratory Pathogenesis, Prevention and Treatment of High Incidence Diseases in Central Asian, First Affiliated Hospital of Xinjiang Medical University, Liyushan Road, Urumqi, Xinjiang, 830054, China

2. Yingjisha County Center for Disease Control and Prevention Kashgar Region, Xinjiang, 844500, China

3. Kashgar Regional Lung Hospital, Kashgar, 844000, China

4. Urumqi Municipal Center for Disease Control and Prevention, 830026, China

5. High-tech District Center for Disease Control and Prevention of Urumqi, Xinjiang, 830002, China

6. Xinhuarui Information Co., LTD, Urumqi, Xinjiang, 830011, China

\section{Availability of data and materials}

All the data supporting our findings have been presented in this paper.

\section{Consent for publication}

Not applicable.

\section{Competing interests}


The authors declare that they have no competing interests.

\section{References}

1. Zumla A, George A, Sharma V, Herbert RH, Baroness Masham of I, Oxley A, Oliver M: The WHO 2014 global tuberculosis report-further to go. Lancet Glob Health 2015, 3(1):e10-12.

2. Onozaki I, Law I, Sismanidis C, Zignol M, Glaziou P, Floyd K. National tuberculosis prevalence surveys in Asia, 1990-2012: an overview of results and lessons learned. Trop Med Int Health. 2015;20(9):1128-45.

3. National Technical Steering Group of the Epidemiological Sampling Survey for T. Implementing Office of the Epidemiological Sampling Survey for $\mathrm{T}$ : [The prevalence of pulmonary tuberculosis in a national survey across China in 2010]. Zhonghua Jie He He Hu Xi Za Zhi. 2012;35(9):665-8. (in Chinese).

4. World Health Organization. (WHO): TB disease burden. Global Tuberculosis Report 2018. Geneva.

5. Li YH, Qi WG. L Analysis of monthly report of tuberculosis control in Xinjiang in 2005. Endemic Diseases Bulletin. 2006;21(6):29-32. (in Chinese).

6. Yang JM, Jaynes SMHL, Tai XR, Li YH, Zhao Z. Analysis of tuberculosis epidemiological survey conducted in 2010-2011 in Xinjiang Uygur Autonomous Region. Chin J Antituberc 2013(35):960964. (in Chinese).

7. Zhao Z, Liu NQ, Yipaer A, Wang XJ. Epidemiological and spatial distribution characteristics of tuberculosis in Xinjiang Uygur Autonomous Region from 2008 to 2018. Chin J Antituberc. 2019;41(8):893-99..(in Chinese).

8. Huang R, Li XY, Xiao H, Tian HY. Application of spatial statistical analysis in tuberculosis study. Chin J Antituberc. 2019;38(6):432-35..(in Chinese).

9. World Health Organization (WHO). Country profiles for 30 high TB burden countries. Global tuberculosis report 2019.

10. World Health. Organization (WHO): Pursue high-quality DOTS expansion and enhancement. 2007.

11. Wang L, Zhang H, Ruan Y, Chin DP, Xia Y, Cheng S, Chen M, Zhao Y, Jiang S, Du X, et al. Tuberculosis prevalence in China, 1990-2010; a longitudinal analysis of national survey data. Lancet. 2014;383(9934):2057-64.

12. Wang XQ, Li YH, Ipal A. Endemic situation and prevention and control of tuberculosis in Kashi, Xinjiang from 2005 to 2009. Bulletin of Infectious Disease Control 2011(03):10-12. (in Chinese).

13. National Health and Family Planning Commission of the People's Republic of China. WS288-2017 Diagnosis for pulmonary tuberculosis[S]. Beijing: China Standard Press; 2018. pp. 1-24. (in Chinese).

14. World Health Organization. (WHO): Treatment of Tuberculosis: Guidelines. Fourth Edition. 2010.

15. Technical Guidance Group of the Fifth National TB Epi-demiological Survey;The Office of the Fifth National TB Epidemiological Survey. The fifth national tuberculosis epidemiological survey in 2010. 
Chinese Journal of Antituberculosis. 2012;34(8):485-508. (in Chinese).

16. Nahid P, Jarlsberg LG, Rudoy I, de Jong BC, Unger A, Kawamura LM, Osmond DH, Hopewell PC, Daley $\mathrm{CL}$. Factors associated with mortality in patients with drug-susceptible pulmonary tuberculosis. $\mathrm{BmC}$ Infect Dis 2011, 11.

17. Xu WG, Lu W, Zhou Y, Zhu LM, Shen HB, Wang JM. Adherence to anti-tuberculosis treatment among pulmonary tuberculosis patients: a qualitative and quantitative study. Bmc Health Serv Res 2009, 9.

18. Rocha M, Pereira S, Ferreira L, Barros H. The role of adherence in tuberculosis HIV-positive patients treated in ambulatory regimen. Eur Respir J. 2003;21(5):785-8.

19. Yao S, Huang WH, van den Hof S, Yang SM, Wang XL, Chen W, Fang XH, Pan HF. Treatment adherence among sputum smear-positive pulmonary tuberculosis patients in mountainous areas in China. BMC Health Serv Res. 2011;11:341.

20. Tang Y, Zhao MG, Wang YX, Gong YH, Yin XX, Zhao AG, Zheng JJ, Liu ZY, Jian XF, Wang WX, et al: Non-adherence to anti-tuberculosis treatment among internal migrants with pulmonary tuberculosis in Shenzhen, China: a cross-sectional study. Bmc Public Health 2015, 15.

21. Gong XJ, Li YH, Wang J, Wu G, Mohemaiti A, Wushouer Q, Yao LD, Lv JH, Li RR, Li PF, et al. Treatment adherence among sputum smear-positive pulmonary tuberculosis patients in Xinjiang, China: a prospective study. Rsc Adv. 2018;8(16):8983-9.

22. Kaona FA, Tuba M, Siziya S, Sikaona L. An assessment of factors contributing to treatment adherence and knowledge of TB transmission among patients on TB treatment. BMC Public Health. 2004;4:68.

23. Driver CR, Matus SP, Bayuga S, Winters Al, Munsiff SS. Factors associated with tuberculosis treatment interruption in New York City. J Public Health Manag Pract. 2005;11(4):361-8.

24. Sabaté E. Adherence to long-term therapies. Geneva: World Health Organization; 2003.

25. Morsky D, Malotte C, Choi P. A patient education programme to improve adherence rates with antituberculosis drug regimens. Health Educ Q. 1999;17:253-67.

26. World Health Organization (WHO): The Stop TB Strategy. 2006, Geneva, Switzerland, WHO/HTM/TB/2006.368.

\section{Figures}




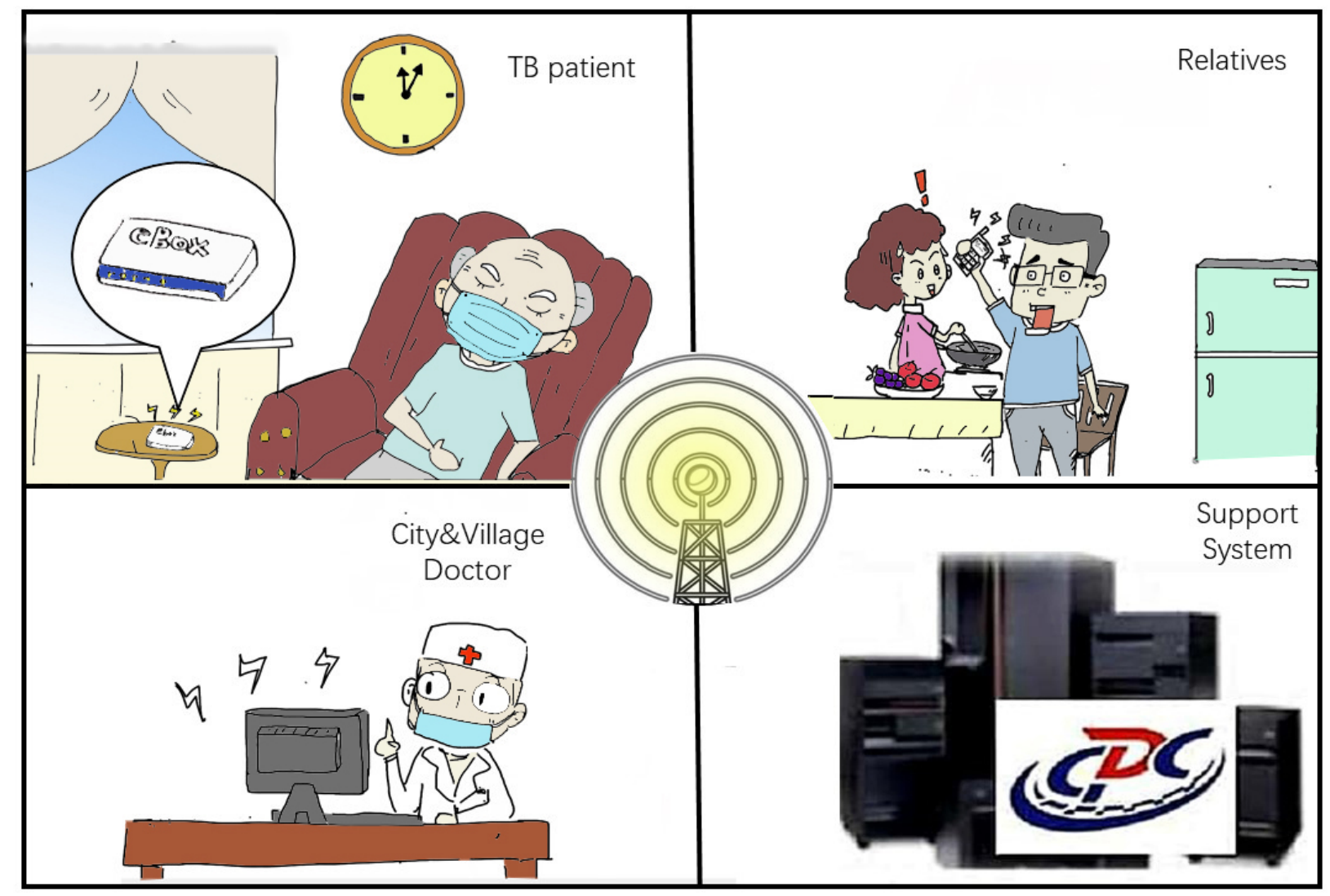

Figure 1

Working flow diagram of eDOTS system

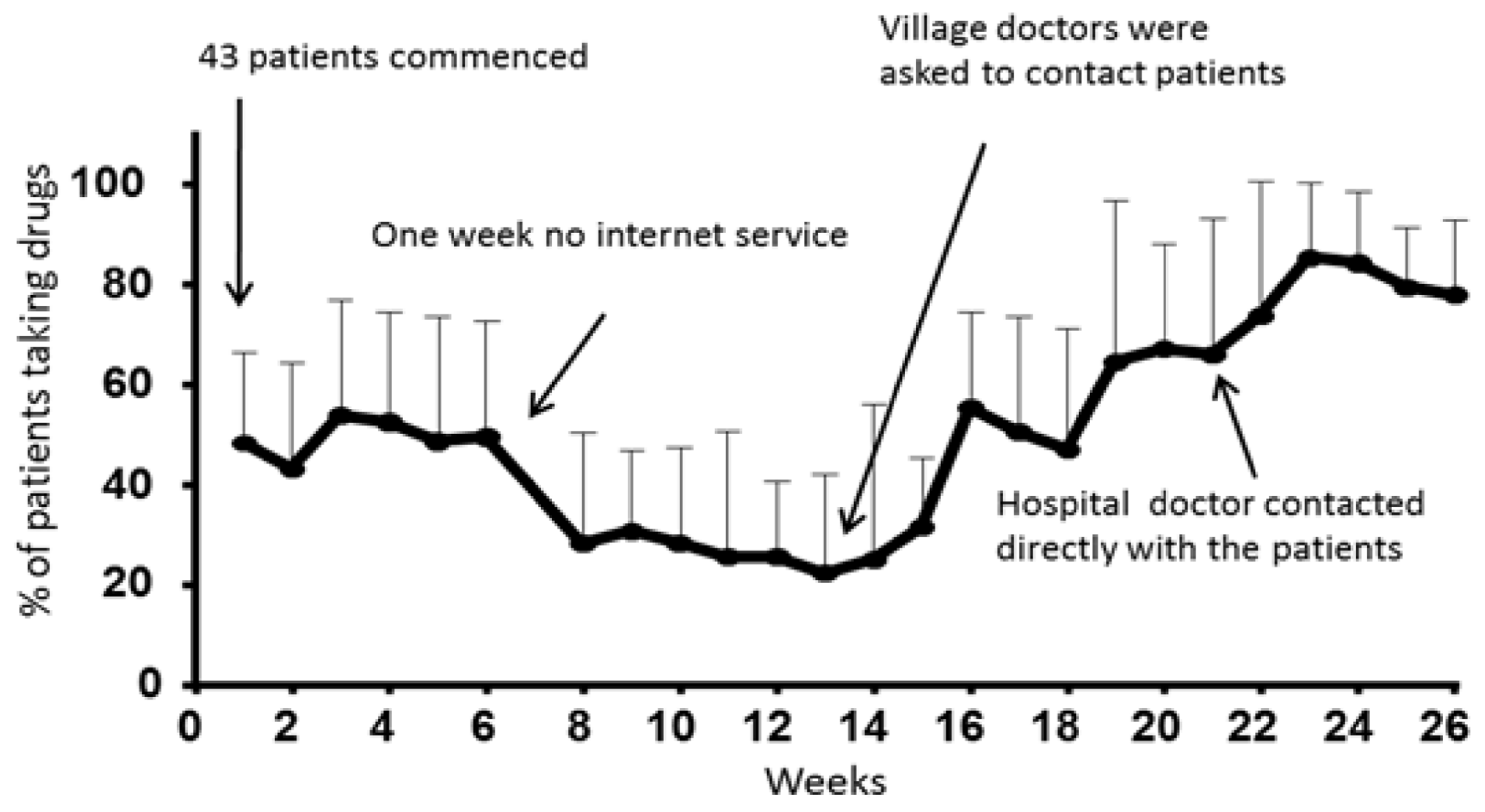

Figure 2 
The mean percentage of village patients taking antibiotics weekly recorded by eDOTS

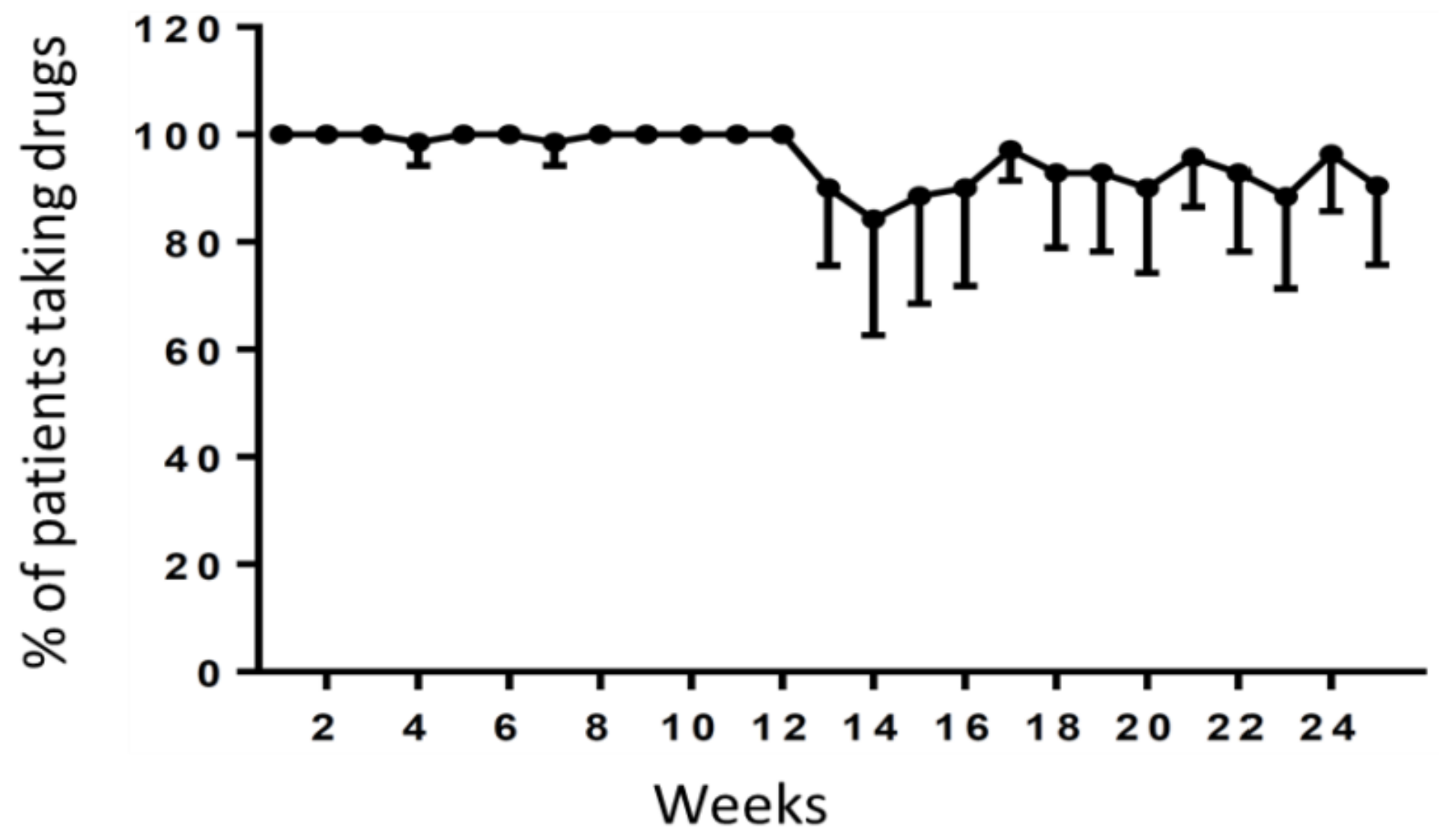

Figure 3

The mean percentage of township patients taking antibiotics weekly recorded by eDOTS 


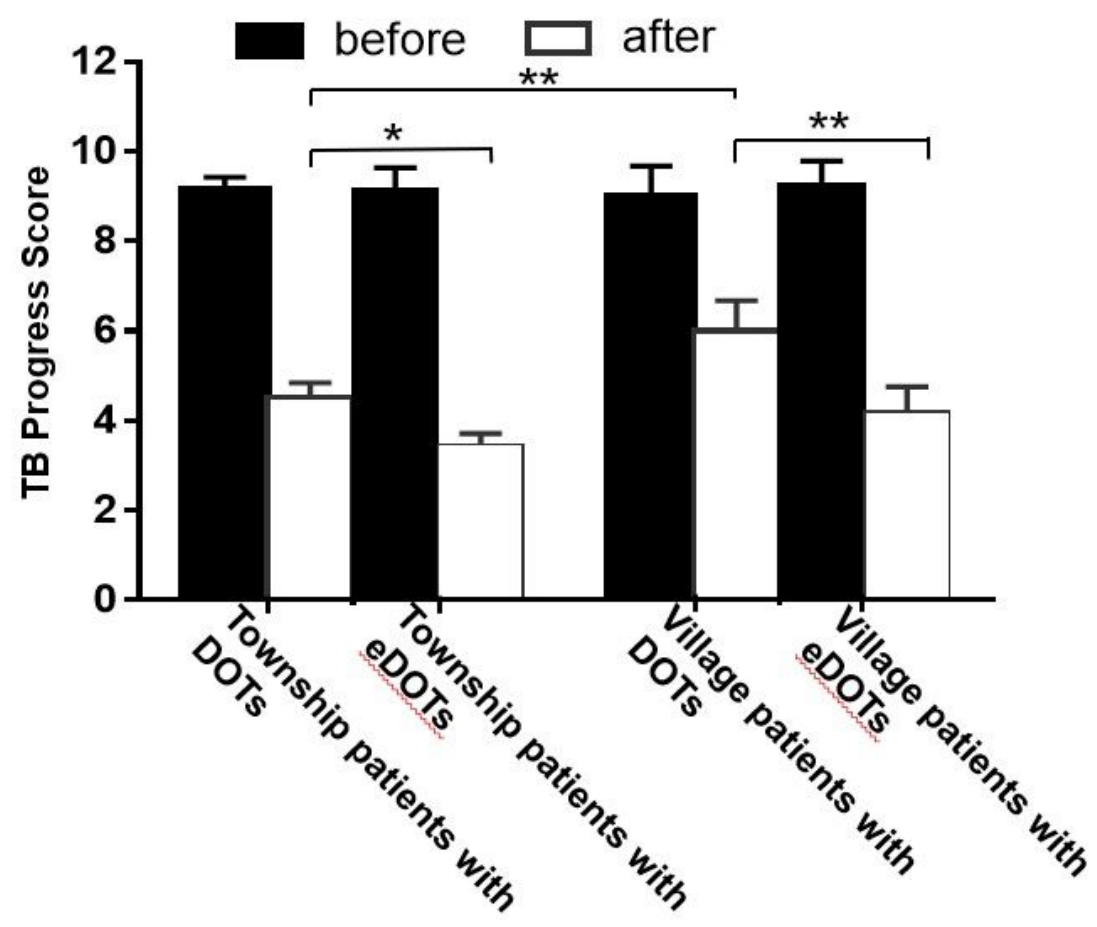

Figure 4

Comparison of treatment efficacy of patients with eDOTS with these patients treated with DOTS

\section{Supplementary Files}

This is a list of supplementary files associated with this preprint. Click to download.

- Fig.S2ExamplesofscoringTBlesion.jpg

- Fig.S1Divisionoflungs.jpg 